\title{
ADVANCED TOOLS FOR FAST MICRO-MODELLING OF MASONRY STRUCTURES
}

\author{
M. PETRACCA ${ }^{1 *}$, C. MARANO ${ }^{2}$, G. CAMATA ${ }^{2}$, E. SPACONE ${ }^{2}$, AND L. PELÀ ${ }^{3}$ \\ ${ }^{1}$ ASDEA Software \\ Via Breviglieri, 8 - 65128 Pescara, Italy \\ e-mail: m.petracca@asdea.net, https://asdeasoft.net (*corresponding author) \\ ${ }^{2}$ Università degli Studi G. d'Annunzio Chieti e Pescara \\ Department of Engineering and Geology (InGeo) \\ Viale Pindaro, 42 - 65127 Pescara, Italy \\ email: \{corrado.marano,guido.camata\}@unich.it \\ ${ }^{3}$ Universitat Politècnica de Catalunya (UPC-BarcelonaTech) \\ Department of Civil and Environmental Engineering \\ Jordi Girona 1-3, 08034 Barcelona, Spain \\ e-mail: luca.pela@upc.edu
}

Keywords: Masonry, Micro-Modelling, Continuum Damage, IMPLEX

\begin{abstract}
Among all the approaches commonly used to study masonry structures, micromodelling is the most accurate. Masonry can be seen as a composite material made of bricks and mortar joints. Their different mechanical properties, their geometry and their arrangement inside the micro-structure, lead to very complex behaviours that are often difficult to represent using equivalent homogenous constitutive models commonly available in commercial and research FEM solvers. Micro-modelling can capture the complex non-linear behaviours of masonry by explicitly modelling the micro-structure inside the computational model. Micromodelling leads to models with a large number of finite elements, thus increasing prohibitively the computational time. This is also due to the problem of solving complex nonlinear solutions involving damage and strain localization, leading to very small time-steps required to achieve convergence. Another issue with micro-modelling is the increased complexity in generating the finite element mesh with all the details of the micro-structure. This work presents some advanced tools that can decrease the high computational time required by micromodelling. A 2-parameter tension-compression plastic-damage constitutive model is presented as an extension of an existing model previously formulated by some of the authors [1,2,3]. The model is implemented in the open-source FEM code OpenSEES [12] with the IMPL-EX method [5], a mixed implicit-explicit integration method that renders the response of this constitutive model step-wise linear, thus removing the convergence issues typically encountered when dealing with softening responses. This research also presents a tool implemented in the STKO (Scientific ToolKit for OpenSees) pre- and post-processor [4] able to automatically convert a homogeneous CAD geometry of a building into a micro-model.
\end{abstract}




\section{INTRODUCTION}

From a structural point of view masonry can be regarded as a composite material made of bricks and mortar joints. Their constitutive response and their spatial arrangement inside the micro-structure, lead to very complex nonlinear behaviours characterized by different failure mechanisms. Many numerical approaches were proposed in the literature to simulate the structural response of complex masonry buildings, including homogenous continuum models [6], detailed micro-models [1,7], and computational homogenization multi-scale models $[2,3,8]$. Among all those approaches, micro-modelling is the one that delivers best accuracy. The main advantage of micro-modelling is its good capability of capturing all the complex failure mechanisms that can arise at the micro-structural scale, such as the damage-induced anisotropy, even when simple isotropic constitutive models are used to model the constitutive behaviour of the microscopic components. On the other side, however, micro-modelling has also some drawbacks that often limit its applicability to the simulation of real-life large-scale structure. When used to model large-scale structures, micro-modelling clearly leads to a very large number of finite elements, and consequently to very expensive analyses, both in terms of time and computational power. Furthermore, the presence of constitutive models exhibiting damage growth and strain localization poses the extra issue of poor convergence even when small time steps are used. Last but not least, there is the problem of preparing the geometric model of an entire structure with all the details of the micro-scale. All the aforementioned drawbacks can be however mitigated to a good extent using the right tools, such as parallel

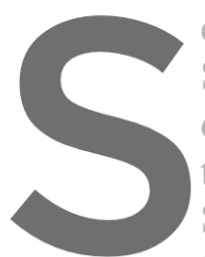
computing, special inte Section 2 describes brie damage model previous the implementation of th Section 4 briefly present OpenSEES that allows the end-user to automatically generate

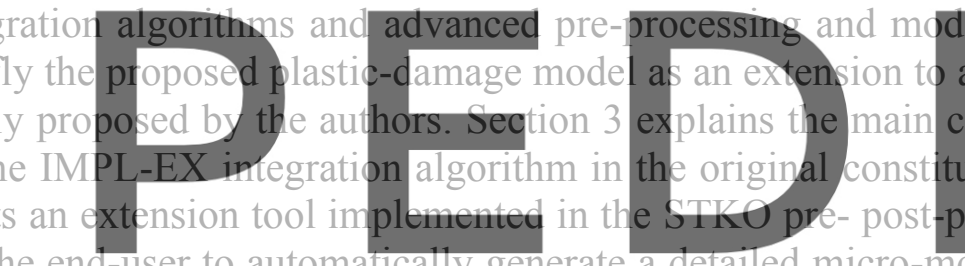
from a homogeneous surface model of the structure. Finally, Section 5 shows a numerical

\section{TENSION-COMPRESSION PLASTIC-DAMAGE MODEL}

This section briefly explains the formulation of the proposed tension-compression plasticdamage model, which is an extension to a tension-compression damage model previously formulated by the authors. For more details about the original damage model the user can refer to [1]. In the following, all variables without subscript refer to the current time-step, while those with the $n$ and $n-1$ subscripts refer to the same variables at the two previous (known) time steps.

The $d^{+} / d^{-}$plastic-damage model, is based on the $d^{+} / d^{-}$pure damage model described in [1], with the addition of plasticity. Note however that the main aim of this work is to have a simple and robust constitutive model that accounts for plasticity just as an overall process involving irreversible deformations for a better description of the cyclic behaviour of masonry. For this reason, the typical ingredients of the classical theory of plasticity are not included in the present formulation. Instead, this model recasts the computation of plastic deformations in the same framework of classical continuum damage models.

The nominal stress tensor $\boldsymbol{\sigma}$ is defined as 


$$
\boldsymbol{\sigma}=\left(1-d^{+}\right) \overline{\boldsymbol{\sigma}}^{+}+\left(1-d^{-}\right) \overline{\boldsymbol{\sigma}}^{-}
$$

where $\overline{\boldsymbol{\sigma}}^{+}$and $\overline{\boldsymbol{\sigma}}^{-}$are the positive and negative parts of the effective stress tensor, while $d^{+}$and $d^{-}$are the positive and negative cracking damage variables, that account for stress reduction and stiffness degradation from the effective stress space, due to opening of cracks. The effective stress tensor $\overline{\boldsymbol{\sigma}}$ is defined as

$$
\begin{gathered}
\overline{\boldsymbol{\sigma}}=\overline{\boldsymbol{\sigma}}^{+}+\overline{\boldsymbol{\sigma}}^{-} \\
\overline{\boldsymbol{\sigma}}^{+}=\left(1-d_{p l}{ }^{+}\right) \widetilde{\boldsymbol{\sigma}}^{+} ; \overline{\boldsymbol{\sigma}}^{-}=\left(1-d_{p l}{ }^{-}\right) \widetilde{\boldsymbol{\sigma}}^{-}
\end{gathered}
$$

where $\widetilde{\boldsymbol{\sigma}}^{+}$and $\widetilde{\boldsymbol{\sigma}}^{-}$are the positive and negative parts of the trial effective stress tensor $\widetilde{\boldsymbol{\sigma}}$, while $d_{p l}{ }^{+}$and $d_{p l}{ }^{-}$are the positive and negative plastic damage variables, that account for stress reduction of the trial effective stress due to plastic flow. The trial effective stress tensor $\widetilde{\sigma}$ is defined as

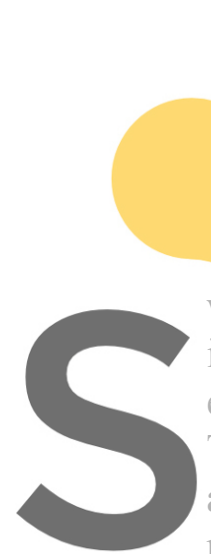

$$
\begin{gathered}
\widetilde{\sigma}=\bar{\sigma}_{n}+C:\left(\varepsilon-\varepsilon_{n}\right) \\
\widetilde{\sigma}^{+}=\sum_{i=1}^{3}\left\langle\tilde{\sigma}_{i}\right\rangle p_{i} \otimes p_{i} \\
\widetilde{\sigma}^{-}=\widetilde{\sigma}-\widetilde{\sigma}^{+}
\end{gathered}
$$

\section{where $\bar{\sigma}_{n}$ is the effective stress (i.e. only including plasticity) at the previous time ster}

is the elastic (trial) eigenvector.

The evolutions of plastic an evolution of the plast negative failure criteria, or darnage surfaces, are similar
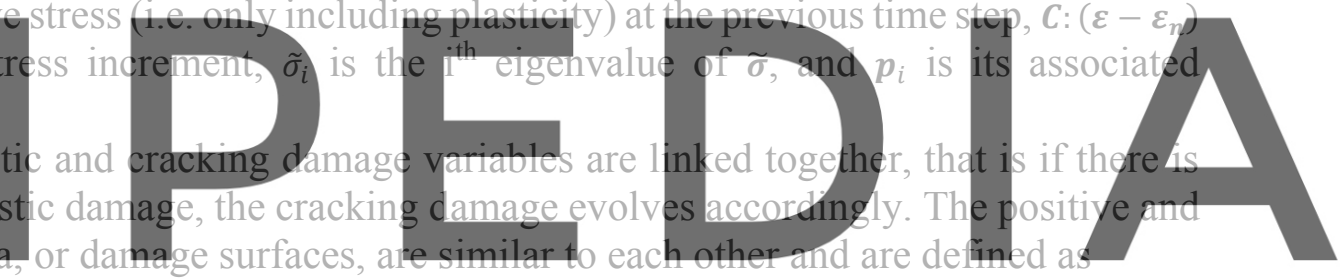

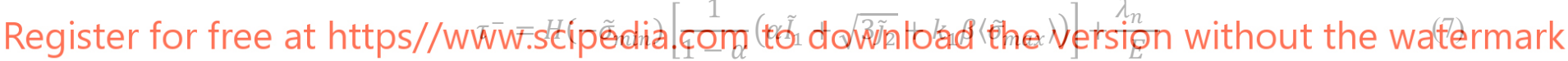

$$
\tau^{+}=H\left(\tilde{\sigma}_{\text {max }}\right)\left[\frac{1}{1-\alpha}\left(\alpha \tilde{I}_{1}+\sqrt{3 \tilde{J}_{2}}+\beta\left\langle\tilde{\sigma}_{\text {max }}\right\rangle\right) \frac{f_{t}}{f_{c p}}\right]+\frac{\lambda_{n}{ }^{+}}{E}
$$

$\tau^{+}$and $\tau^{-}$are the so-called equivalent (scalar) positive and negative stresses. The reader can refer to [1] for a more detailed description of each variable that appear the two failure criteria. The Heaviside functions of the minimum and maximum eigenvalues is needed to activate the compressive surface only if at least one eigenvalue is negative, and, accordingly, the tensile surface only if at least one eigenvalue is positive. This is required to avoid tensile damage growth under purely compressive stress states and to avoid compressive damage growth under purely tensile stress states. In fact, even if the positive and negative damage variables affect only the associated positive and negative parts of the stress, their failure surfaces are function of the whole stress tensor.

The last terms in Equations 7 and 8 are functions of the Young's modulus $E$ and the positive and negative equivalent plastic strains $\lambda_{n}{ }^{+}$and $\lambda_{n}{ }^{-}$known from the previous time step. These two last terms where not present in the original pure damage model in [1]. In fact, here, due to plasticity, $\tau^{+}$and $\tau^{-}$are function of $\widetilde{\boldsymbol{\sigma}}$, which is not the total stress computed from the total 
strain. The last two terms in Equations 7 and 8 are included to compute $\tau^{+}$and $\tau^{-}$as if they were computed from the total strain. In this way it can be assured that, in loading conditions, $\tau^{+}$and $\tau^{-}$are always larger than their values at the previous time step.

Figure 1 shows the shape of the two damage surfaces in the principal stress space. The compressive surface is represented for different values of the $k_{1}$ parameter.

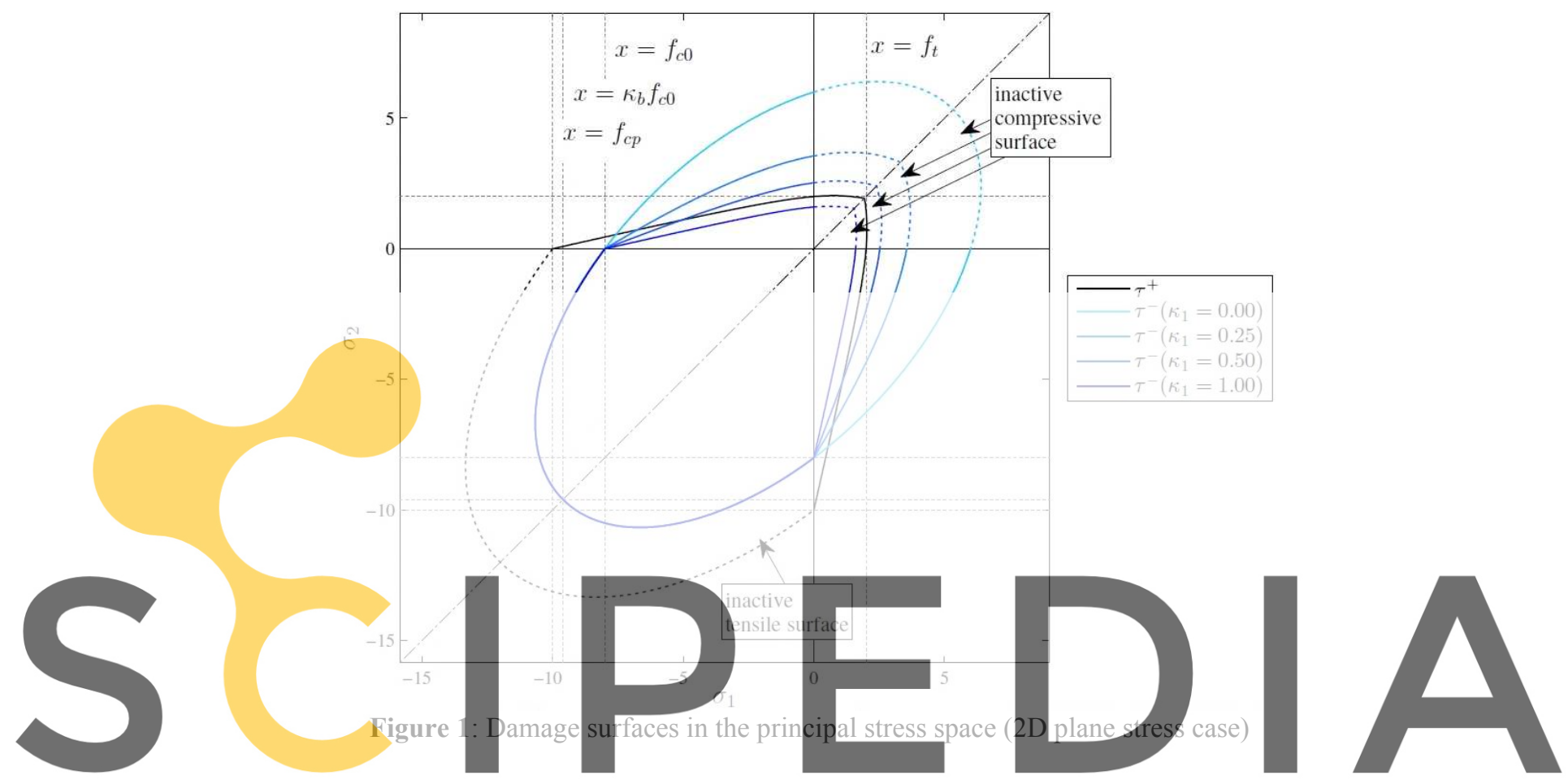

Being the damage and irreversible process, the model introduces the so-called damage

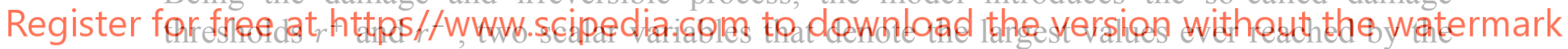

equivalent stresses $\tau^{+}$and $\tau^{-}$during the entire loading history for each time step $t$.

$$
\begin{aligned}
& r^{+}(t)=\max \left(\max _{s \in[0, t]} \tau^{+}(s) ; f_{t}\right) \\
& r^{-}(t)=\max \left(\max _{s \in[0, t]} \tau^{-}(s) ; f_{c 0}\right)
\end{aligned}
$$

At this point, in view of the last two terms added in Equations 7 and 8, $r^{+}$and $r^{-}$are two scalar measures of the stress tensor as if they were computed from the total strain. In fact, we can compute their total-strain counterparts, and the associated nominal (including the effect of both plasticity and damage) hardening variables, as

$$
\begin{gathered}
\varepsilon_{t o t}{ }^{\mp}=\frac{r^{\mp}}{E} \\
q^{\mp}=H^{\mp}\left(\varepsilon_{t o t}{ }^{\mp}\right)
\end{gathered}
$$

The hardening curves function of $\varepsilon_{t o t}{ }^{\mp}$ are reported in Figure 2. For a detailed description the reader can refer to [1]. 


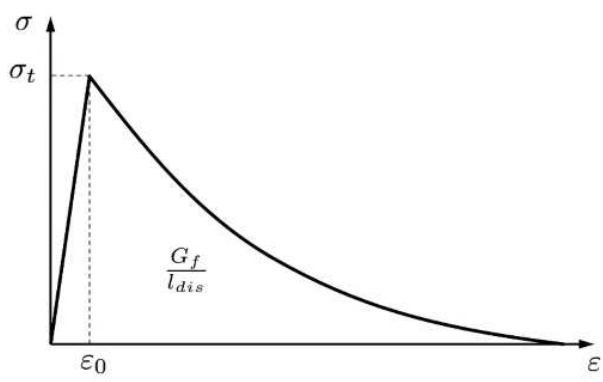

(a)

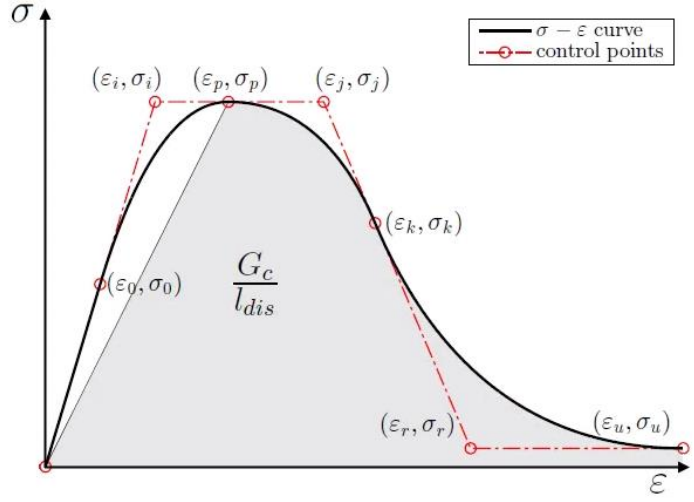

(b)

Figure 2: Tensile (a) and compressive (b) hardening functions

The positive and negative plastic damage variables can be computed as

$$
d_{p l}{ }^{\mp}=1-\frac{q_{p l}{ }^{\mp}}{\tilde{r}^{\mp}}
$$

$$
\tilde{r}^{\mp}=E\left(\varepsilon_{t o t}{ }^{\mp}-\lambda_{n}{ }^{\mp}\right)
$$
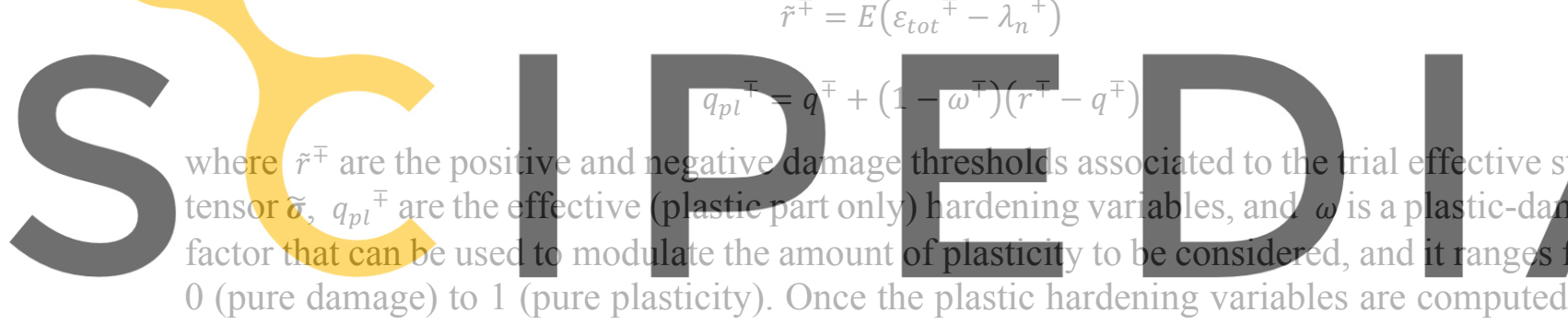

$$
d^{\mp}=1-\frac{q^{\mp}}{q_{p i}{ }^{\mp}}
$$

When both plastic and cracking damage variables are computed, the plastic effective stress tensor and the nominal (damaged) stress tensor can be calculated as per Equations (3) and (1) respectively, and the equivalent plastic strains can be updated as

$$
\lambda^{\mp}=\varepsilon_{t o t}{ }^{\mp}-\frac{q_{p l}{ }^{\mp}}{E}
$$

\section{IMPL-EX ALGORITHM}

It is well known that nonlinear constitutive models exhibiting strain softening response lead to instabilities and the convergence of the iterative procedure may not be achieved at all. To improve the stability of this kind of analyses, the so called IMPL-EX algorithm originally formulated in [5] was implemented in the proposed tension-compression plastic-damage model. The IMPL-EX algorithm is a mixed implicit/explicit procedure to integrate the constitutive 
response. The main idea is that the computation of the constitutive model is divided in two main stages:

Explicit integration. During the iterative procedure the damage thresholds $r^{+}$and $r^{-}$ at time step $t_{n+1}$ are extrapolated from the previous values at $t_{n-1}$ and $t_{n}$ which are known from previous time steps as

$$
r_{n+1}^{\mp}=r_{n}^{\mp}+\frac{\Delta t_{n+1}}{\Delta t_{n}}\left(r_{n}^{\mp}-r_{n-1}^{\mp}\right)
$$

In this way the solution depends only linearly on the current strain. However, there is another source of nonlinearity, that is the spectral decomposition used to compute the positive and negative parts of the trial effective stress tensor. To avoid this source of nonlinearity, the projection tensors $\boldsymbol{P}^{+}$and $\boldsymbol{P}^{-}$(as defined in [10]) used to compute the positive and negative parts of the trial stress tensor are those of the previous time step.

- Implicit correction. After the equilibrium is achieved the standard implicit evaluation of the internal variables is carried out. In this way this second step can be seen as an implicit correction to the previously described explicit extrapolation.

\section{STKO USER INTERFACE AND AUTOMATIC MICRO-MODEL GENERATION}

It is well known that one of the limitations of micro-modelling is the excessively high computational time, due to the large number of finite elements required to represent the microstructure all over the str element solver is not th real-life structure can be challenging. In fact, $m$ modelling of masonry, focus on simple shear-w pre-processor is used. TH
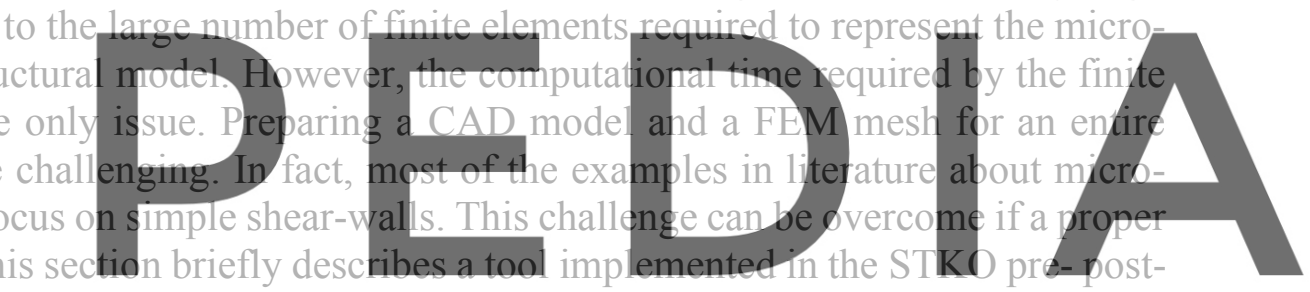

processor for OpenSEES [4], that allows the analyst to convert a simple 2D or 3D model made

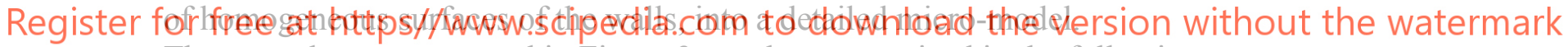

The procedure, represented in Figure 3, can be summarized in the following steps:

User imput. The analyst draws all the surfaces, planar or curves, of the walls of the entire structure, taking care of including holes for the openings. Further, the analyst draws a sample (a repeating unit) of the micro-structure, already equipped with the constitutive models for all micro-structural components (bricks and mortar joints).

- Reparameterization. Each generic 3D surface of the structure can be arbitrarily oriented in the space, and does not need to be necessarily planar. Therefore, each surface will be automatically remapped to a 2D parametric space.

- $\quad$ Fill. Each surface in the 2D parametric space is filled with the repeating unit of the micro-structure.

- Cut. All the pieces of the micro-structure falling into the openings are cut and removed by means of Boolean operation

- Details. If necessary, extra details such as arches or lintels on top of the openings are added based on simple user-defined parameters.

- $\quad$ Remapping. Finally, each surface filled with the micro-structure is remapped to the 3D space and assembled with the other surfaces of the model. 


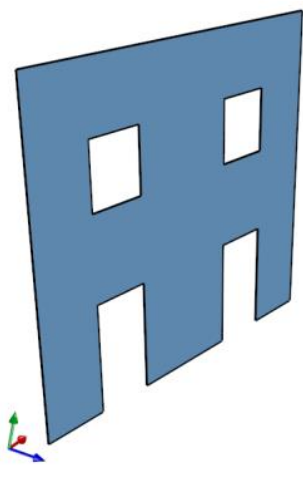

(a)

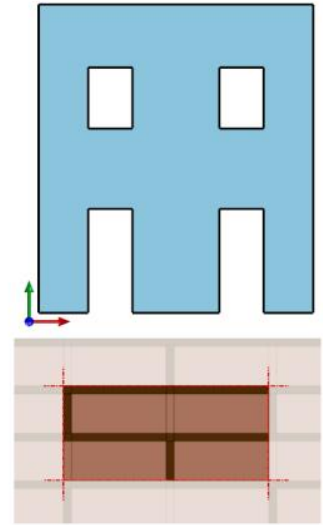

(b)

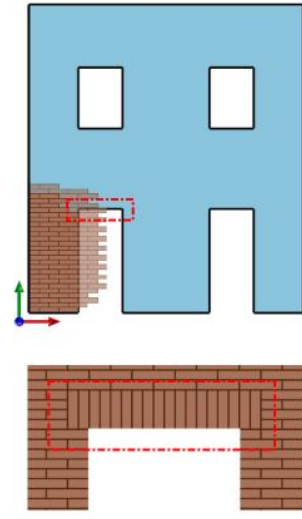

(c)

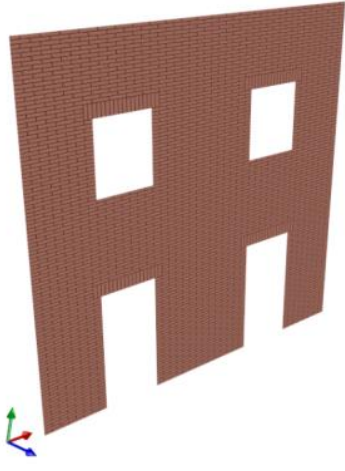

(d)

Figure 3: (a) User input as a homogeneous surface; (b) Reparameterization and micro-sample; (c) Fill, Cut and Details; (d) Remapping to the 3D domain

\section{NUMERICAL APPLICATION}

In this section a numerical application of the proposed plastic-damage model is presented. A two-story masonry building tested at the university of Pavia, and described in detail in [11], is used and simulated numerically to assess the effectiveness of the proposed plastic-damage model. The numerical model was created in STKO pre-post-processor [4], which automatically produced the TCL input files for the OpenSEES MP parallel solver. The results were obtained from the MPCO Recorder inplemented by some of the authors in OpenSEES [12], and

visualized in the STKO post-processor. Figure 4 shows a scherne of the tested prototype. Equal loads are applied at the floor levels along the two lateral valls (Door Wal and Window Wall). Since the Door Wall is not connected to the rest of the structure, it was analyzed separately in Register for Preedel.https//www.scipedia.com to download the version without the watermark

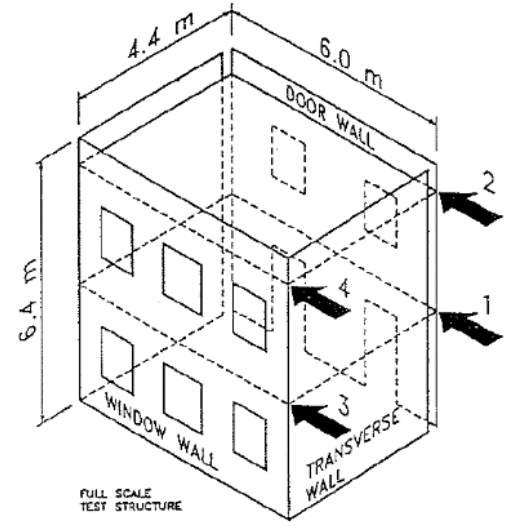

(a)

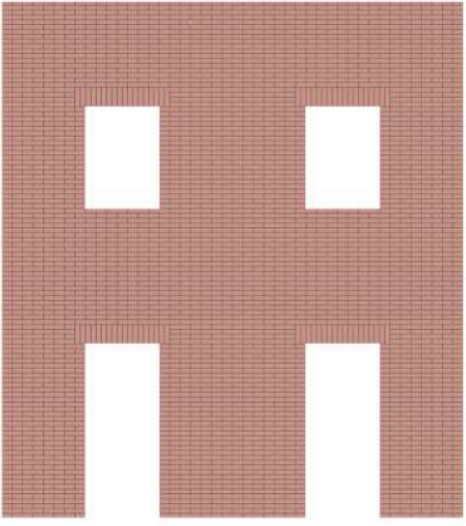

(b)

Figure 4: (a) Scheme of the tested building, taken from [11], and (b) numerical micro-model of the Door Wall 
Table 1 shows the plastic-damage material parameters used for bricks and mortar joints. Figure 5 shows the obtained load displacement-curve and Figure 6 shows the final crack pattern compared with the one obtained from the experimental test. The results are quite good in terms of maximum strength, crack pattern and cyclic hysteresis loop.

Table 1: Constitutive model parameters for bricks and mortar joints

\begin{tabular}{|c|c|c|c|c|}
\hline PARAMETER & DESCRIPTION & UNIT & MORTAR & BRICK \\
\hline $\mathrm{E}$ & Young's modulus & $\mathrm{N} / \mathrm{mm}^{2}$ & 400.0 & 2500.0 \\
\hline$v$ & Poisson's ratio & - & 0.18 & 0.18 \\
\hline $\mathrm{ft}$ & Tensile strength & $\mathrm{N} / \mathrm{mm}^{2}$ & 0.15 & 1.6 \\
\hline $\mathrm{Gt}$ & Tensile fracture energy & $\mathrm{N} / \mathrm{mm}$ & 0.04 & 0.03 \\
\hline$f c p$ & $\begin{array}{c}\text { Compressive strength at } \\
\text { peak }\end{array}$ & $\mathrm{N} / \mathrm{mm}^{2}$ & 5.0 & 26.0 \\
\hline$\varepsilon \mathrm{cp}$ & $\begin{array}{c}\text { Compressive } \\
\text { deformation at peak }\end{array}$ & - & 0.03 & 0.02 \\
\hline & $\begin{array}{c}\text { Compressive fracture } \\
\text { energy }\end{array}$ & $\mathrm{N} / \mathrm{mm}$ & 80.0 & 20.0 \\
\hline
\end{tabular}
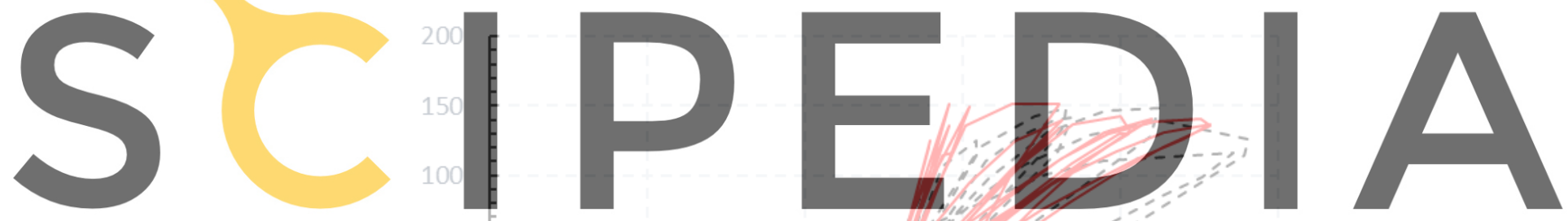

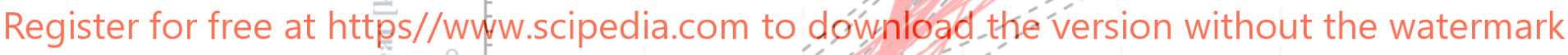

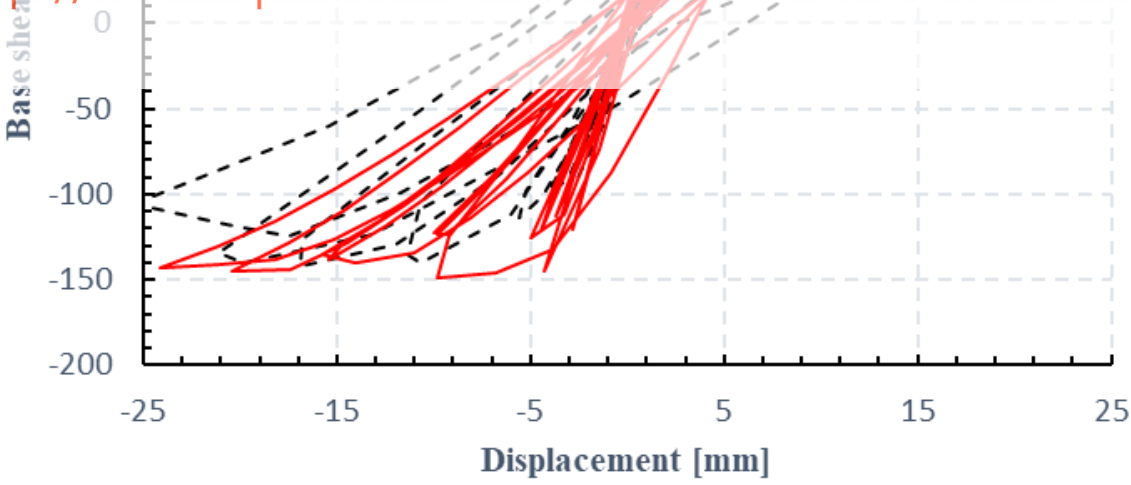

Figure 5: Load-displacement curve for the Door Wall 


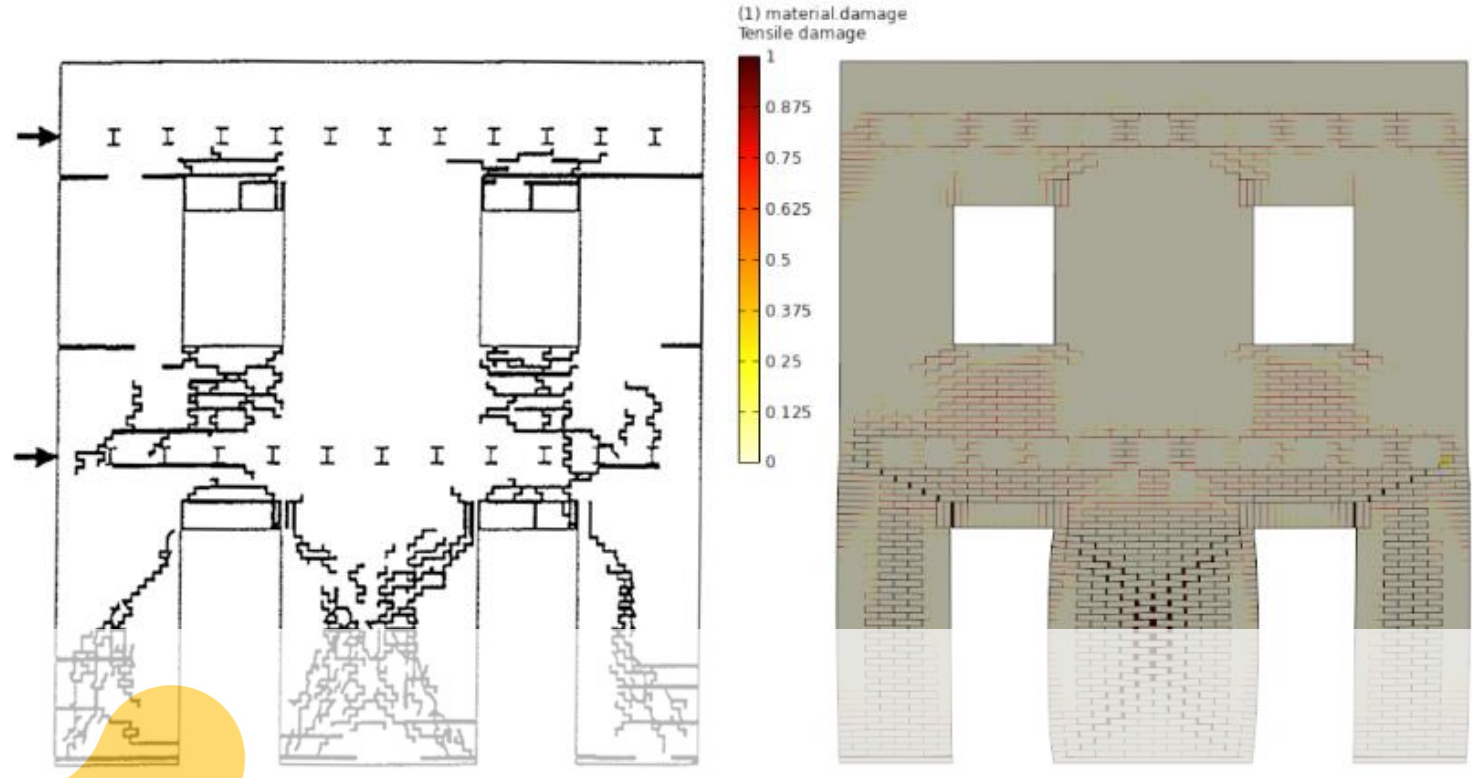

Figure 6: Comparison of the crack pattern at the end of the analysis

6. COMPUTATIONAL COSTS

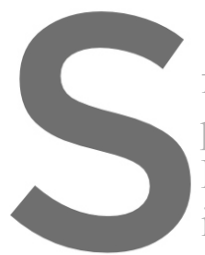

The entire mesh cons fully-integrated planф-stress partitioned in 6 sub-don Intel Core $17-7700 \mathrm{~K} 4.2$ in less than 25 minutes $20 \mathrm{GHz}$
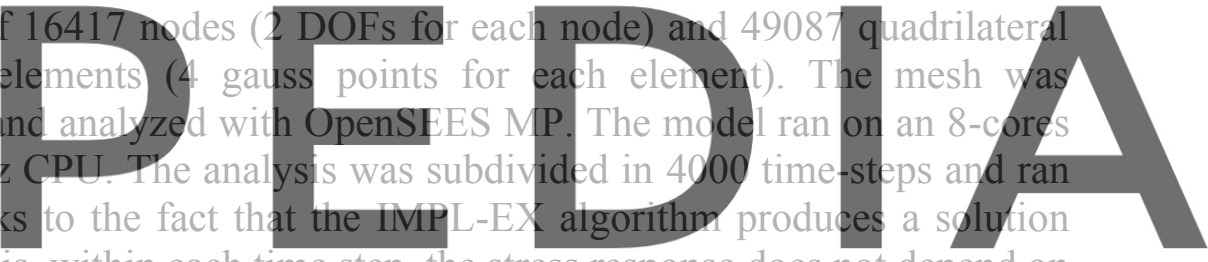

which is step-wise linear, that is, within each time step, the stress response does not depend on

Register for frial strain.

\section{CONCLUSIONS}

- $\quad$ Micro-modelling is indeed an accurate approach for evaluating the complex nonlinear response of materials such as masonry with a very heterogenous micro-structure where each constituent exhibit highly nonlinear responses. The explicit modelling of the micro-structure captures very well the complex geometric interactions and failure mechanisms that arise at the micro-scale level.

- Unfortunately, the computational cost becomes excessively high, and can drastically increase when standard implicit algorithms are used to solve equilibrium due to the brittle nature of the masonry constituents.

- However, if the right tools are used, the aforementioned drawbacks can be satisfactorily mitigated. Parallel computing offers the first speed up, partitioning the entire domain in many smaller sub-domains that can be analyzed in parallel. The IMPL-EX algorithm removes the strong nonlinearity given by the plastic-damage model, thus improving the convergence, and consequently reducing the computation time. Finally, the burden of creating a complex CAD model such as the micro-model shown in this work, can be overcome using dedicated user interfaces such as STKO. 


\section{REFERENCES}

[1] Petracca, M., Pelà L., Rossi R., Zaghi S., Camata G. and Spacone E. Micro-scale continuous and discrete numerical models for nonlinear analysis of masonry shear walls. Construction and Building Materials (2017), 149:296-314.

[2] Petracca, M., Pelà L., Rossi R., Oller S., Camata G. and Spacone E. Regularization of first order computational homogenization for multiscale analysis of masonry structures. Computational Mechanics (2016) 57:257-276.

[3] Petracca, M., Pelà L., Rossi R., Oller S., Camata G. and Spacone E. Multiscale computational first order homogenization of thick shells for the analysis of out-of-plane loaded masonry walls. Computer Methods in Applied Mechanics and Engineering (2017) 315: $273-301$.

[4] STKO Scientific ToolKit for OpenSees. https://asdeasoft.net/stko/

[5] Oliver, J., Huespe, A. E., and Cante, J. C. An implicit/explicit integration scheme to increase computability of non-linear material and contact/friction problems. Computer Methods in Applied Mechanics and Engineering (2008), 197: 1865-1889.

[6] Pelà, L., Cervera, M., and Roca, P., Continuum damage model for orthotropic materials: Application to masonry, Computer Methods in Applied Mechanics and Engineering (2011), 917-930

[7] Lourenço, P. B., and Rots, J. G. Multisurface interface model for analysis of masonry structures. Journal of engineering mechanics (1997), 123:660-668.

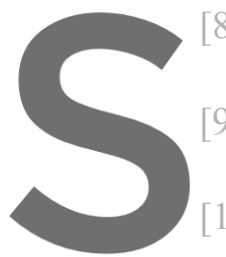
structures. International Journal for Multiscale Computati
Cervera, M., Oliver, J., and Faria, R. Seisinic evaluation
damage models. Earthquake engineering \& structural dyn
] Wu, J. Y., Li, J., and Faria, R. An energy release rateconcrete. International Journal of Solids and Structures (2006), 43:583-612

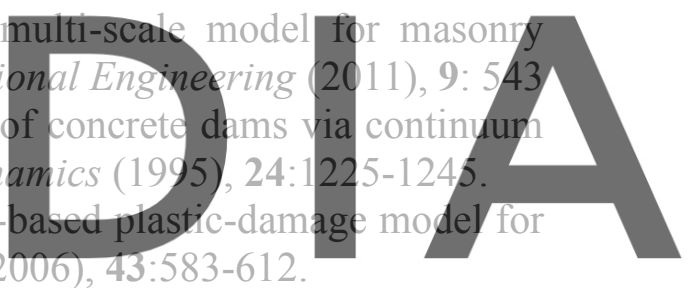

Magenes, G. Calvi, G. M., and Kingsley, G. R. Seismic testing of a full-scale, two-story

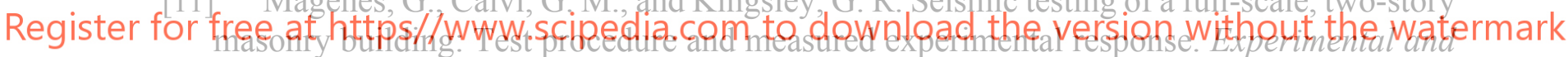
numerical investigation on a brick masonry building prototype - numerical prediction of the experiment, Rep. 3.0. Gruppo Nazionale La Difesa Dai Terremoti, University of Pavia, Pavia, Italy.

[12] McKenna, F. OpenSees: a framework for earthquake engineering simulation. Computing in Science \& Engineering (2011), 13:58-66. 